\title{
Verification of Over-Speed and Burst Margin Limits in Aero Engine Disc along with Low Cycle Fatigue Life
}

\author{
Harinath SP" ${ }^{*}$, Sharath Chandra GV\#, Shreyas PM\# ${ }^{\#}$ and Kumar K Gowda^ \\ *Kshipra simulations Center of excellence and development Banglore-560091, Karnataka, India \\ ${ }^{\wedge}$ Vivekananda Institute of Technology Bangalore - 560074, Karnataka, India \\ Received 02 Jan 2018, Accepted 05 March 2018, Available online 09 March 2018, Vol.8, No.2 (March/April 2018)
}

\begin{abstract}
Aero engine rotor burst evaluation is one of the most important problems to be taken care off, whenever it comes to designing a turbo machinery disc. The consequences of a failure can be intense, since the disc fragments into multiple pieces and they are hurled away in all the possible direction at high speeds. Due to high thermo-mechanical loading conditions the disc is subjected to varying degrees of temperature from bore to rim. However, the centrifugal force dominates in the disc which ranges from 80\%-90\% and the rest can be treated as thermal and gas loads. The challenge lies at designing a disc for off-design conditions with their varying loads and duty cycles. In present work evaluation of safety limits and low-cycle fatigue (LCF) life estimation of an aero engine disc through classical methods and blending the terminologies with simulation engineering to arrive at a probable interpretation of number of duty cycles is carried out. The methodology compares the fatigue parameters involved in evaluation of disc life at off-design condition through sensitivity analysis. The design tool closely connects the flight certification FAA and EASA the regulating agencies for safety in air transportation vehicles. The off-design speed regulations through API and MIL handbook for material specification are considered to carry out design of experiments using finite element analysis approach
\end{abstract}

Keywords: Engine rotor burst, Duty cycles of turbo fan, over-speed and burst margins, low cycle fatigue, Robinson's and Hallinan's failure criteria's, FAA, EASA, Universal slope and Coffin-Manson method.

\section{Nomenclature}

Fc -Centrifugal force in $\mathrm{N}$

$\mathrm{M}$-Mass of the blade in $\mathrm{Kg}$

rc -Center of Gravity in $\mathrm{mm}$

$\omega$ - Angular velocity in rads/s

$\mathrm{N}$-Rotating speed in rpm

$\mathrm{PB}$-Blade pressure in $\mathrm{MPa}$

$\mathrm{Nb}$-Number of blades $=60$

$\mathrm{D}$-Diameter of the disc in $\mathrm{mm}$

$\mathrm{t}$-Thickness of the disc in $\mathrm{mm}$

$\epsilon_{a}$-Strain amplitude

$\epsilon_{f}$-Strain at failure

$\mathrm{Nf}$-Number of cycles

c -Ductility factor

CASE 1- Centrifugal loading only

CASE 2 - Combination of centrifugal force and blade pressure

CASE 3 - Combination of centrifugal, blade pressure and thermal load

\section{Abbreviation \\ API- American Petroleum Institute \\ *Corresponding author's ORCID ID: 0000-0002-4876-1517, DOI: https://doi.org/10.14741/ijcet/v.8.2.10}

ASTM- American Society for Testing Materials Mill handbook - Military handbook standards EASA - European Aviation Safety Agency FAA - Federal Aviation Administrations

\section{Introduction}

Turbo-machinery discs are heavy, highly stressed components used in gas turbines mainly due to overspeed and operating temperatures and these stresses play a key role in failure of disc. The two main international authorities who regulate the safety of air transportation, European Aviation Safety Agency (EASA) [Stefano Chianese,2011] Europe, Federal Aviation Administration (FAA) [Stefano Chianese,2011] USA, have set the requirements to be used for civil air transportation engine components. The disc is made of an isotropic material say INCONEL 718 which is having a particular mechanical property at a specific temperature as per MIL standards. For analysis, duty cycles of turbofan engine are considered which is as shown in figure (1). The failure criteria's to be employed for designing the disc under the safety regulations and applying classical equations to arrive at a thumb rule are a design challenge. Major disc 
design criteria are identified with importance on the life limiting design and the application of life estimating techniques. Design for low-cycle fatigue capability includes consideration of material characteristics, temperature and stress analysis. The evaluation of safety margins and estimation of lowcycle fatigue life in an aero engine disc for design loading conditions (centrifugal force, blade loads and thermal loads) with considering different methods mainly linear and material non-linearity (isotropic and kinematic hardening) is followed. Experimental way of obtaining these safety margins and life of the aero engine disc are very expensive and time consuming. By employing FEA method for evaluating the safety margins and life of disc the above-mentioned problem can be minimized to a great extent. Commercial package software ANSYS is used to carry out the analysis.

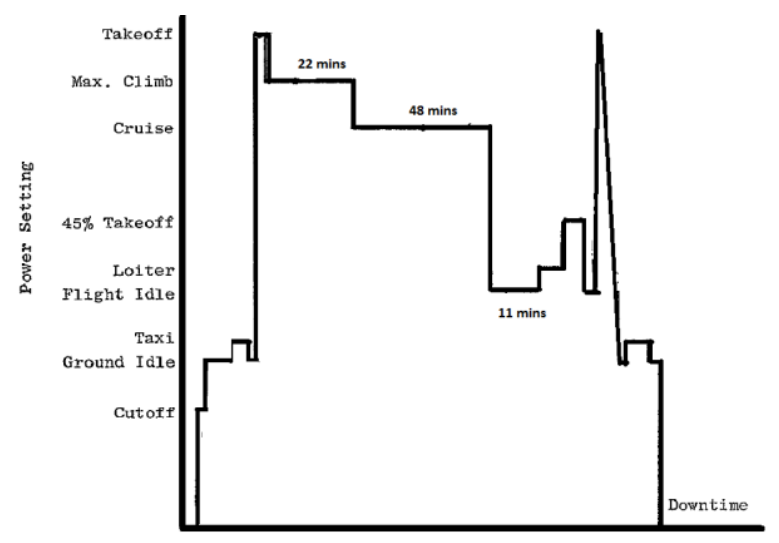

Fig. 1 Duty cycles of GE CF6-50 aero engine [W.N Barack]

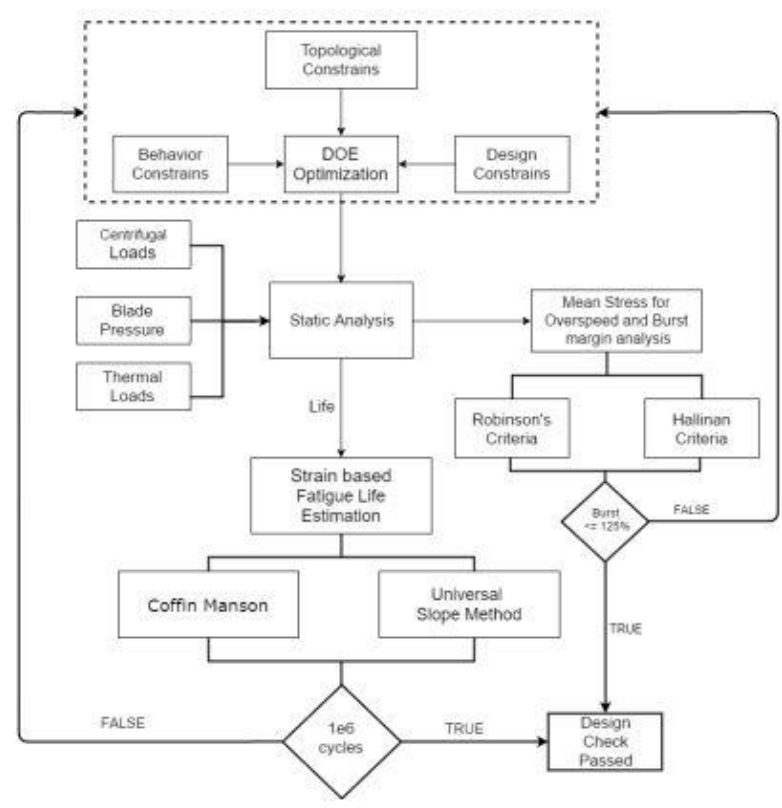

Fig.2 Process for the present work

The safety margins and corresponding speeds are obtained by using two different approaches,
Robinson's and Hallinan's failure criteria's. These two classical equations need to be effectively utilized as per FRC (Flight Readiness Certification). The low-cycle fatigue life is estimated for the disc by considering their respective speeds. By using two methods namely Universal Slope Method and Coffin-Manson Method in this work, the estimation of fatigue life of an aero engine disc is carried out. The above analysis is followed based on the two international agencies (FAA and EASA) which regulate the safety of air transportation. The safety margins obtained for the disc which is subjected to loading conditions mentioned above should follow the API standards and the fatigue life should be greater than $1 * \mathrm{e} 6$ cycles.

The evaluation process of over-speed, burst margin and estimation of fatigue life is as shown in figure (2).

\section{Scope of work}

Gas turbine rotors should pass through design, stress and the quality checks while underdoing certification loads. After the series of checks as per the regulations the major test involves over-speeding, burst evaluation, life estimation, fatigue, creep interaction, fracture and finally the failure analysis. However, hand full of literature is available on individual checks either through mathematical modeling or through experimental work. The modern computers have made it possible to carryout design of experiments employing multi-degrees of freedom analysis carrying multidesign objectives, behavior constraints to achieve design goal. However, the present work is to focus on arriving at optimum disc geometry through DOE considering in-service condition and blend the classical theories with FEA and quickly arrive at commendable methodology which can evolve the design objective in a short duration of time with reliable results and the margin of safety at conservative side.

\subsection{Objectives of present work}

1) Topological optimization to achieve the design goal.

2) A sensitivity study for material model and its behaviour at in-service condition.

3) 3-D analysis for estimation of over-speed and burst margin evaluation

- Hallinans approach

- Robinsons approach

4) Estimation of over-speed margin and the burst speed in rotating aero engine disc as per the international authorities for integrity, blending the classical approach with FEA

5) Estimation of low cycle fatigue life

6) Application of 3-D elasto-plastic strain to classical equations to arrive at fatigue life of disc

- Coffin-Manson method

- Universal slope method 


\section{Load considerations}

\subsection{Rotational velocity Rang}

Rotational or angular velocity is defined as the rate of change of angular displacement and is a vector quantity which specifies the rotational speed (angular speed) of an object and the axis rotation.

\subsection{Influence of the blades}

The presence of blades on the external ring of the disc causes an additional traction load in the radial direction, since they behave like concentrated masses under the effect of the centrifugal field.

Formulas for blade pressure:

$\mathrm{Fc}=\mathrm{m} \omega 2 \mathrm{rc}$

Eqn.1

$\mathrm{PB}=\frac{\mathrm{Fc} * \mathrm{Nb}}{\pi \mathrm{Dt}}$

Eqn.2

\subsection{Thermal load}

Loads due to change in temperature are produced by the non-uniform distribution of temperature in the disc under service conditions. The external region of the rotor that is closer to the hot gasses, in fact, is hotter than the internal area. This non-uniform temperature gradient causes a deformation of the material, along with the coefficient of thermal expansion.

\subsection{Service conditions for disc}

Turbine disc experiences centrifugal loads, thermal loads and blade pressure under operating conditions. The loading conditions of the disc for the present work are classified into three cases: first case is by considering centrifugal force only, second case is combination of centrifugal and blade pressure, third case is by considering all three loads as mentioned above. Figure (3) represents the schematic diagram of disc loading.

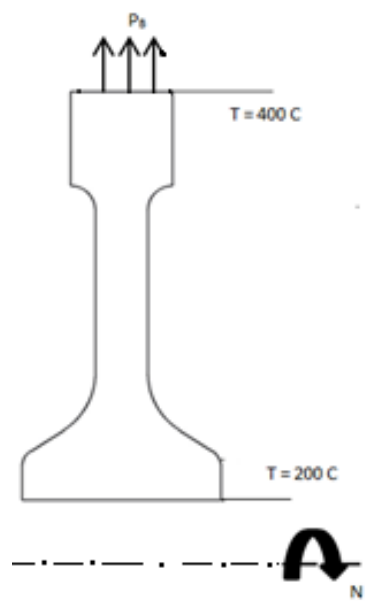

Fig.3 shows the schematic features of the disc

\section{Material}

Inconel alloys are corrosion-resistant materials well suited for service in extreme environments subjected to pressure and heat. Since, the aero engine discs are subjected to high centrifugal and thermal loads Inconel is the material preferred for their manufacturing.

Table 1 shows material property of INCONEL718

\begin{tabular}{|c|c|}
\hline Density & $8190 \mathrm{~kg} / \mathrm{m} 3$ \\
\hline Poison's ratio & 0.3 \\
\hline Young's modulus & $209 \mathrm{GPa}$ \\
\hline Tensile strength & $1035 \mathrm{MPa}$ \\
\hline $\begin{array}{c}\text { Co-efficient of thermal } \\
\text { expansion }\end{array}$ & $13.0 * 10-6 \mathrm{~K}-1$ \\
\hline
\end{tabular}

\section{DOE (Design of Experiments)}

Gas turbine rotors are often subjected to high transient and fluctuating speed loads. Many key parameters from design, material strength, geometry and behavior play a vital role in reliability and robustness during inservice condition. Sensitivity analysis and DOE become very essential to arrive at an optimum geometry. The design parameters and topological constraints considered for the present work are to arrive at optimum disc geometry through DOE which can be broadly classified in sequence

1) Design Parameters

2) Behavior constraints

3) Topological constraints

\subsection{Design parameters}

1) Allowable hoop and von-Mises stress at the bore is $95 \%$ of $0.2 \%$ proof stress.

2) Allowable hoop stress at the web is $85 \%$ of $0.2 \%$ proof stress

3) Allowable radial and von-Mises stress at the web is $80 \%$ of $0.2 \%$ proof stress.

4) Allowable hoop and von Mises stress at the rim is $67 \%$ of $0.2 \%$ proof stress

5) Allowable radial growth $\leq$ specified tolerance of 1 $\mathrm{mm}$

6) Allowable axial growth $\leq$ specified tolerance of 1 $\mathrm{mm}$.

\subsection{Behaviour constraints}

1) The average section stress at cross section of the disc should be within the allowable design limits

2) The average section stress at the cross section should be $10 \%$ less than the blade root neck average stress

3) Allowable AWMHS $<72 \%$ of $0.2 \%$ proof stress at peak temperature in the disc.

4) Allowable AWMHS $<64 \%$ of UTS at peak temperature in the disc. 
5) Disc burst-speed $\geq 125 \%$ of maximum allowable steady state speed for $12000 \mathrm{rpm}$.

6) Disc over-speed $\geq 118 \%$ of maximum allowable steady state speed for $12000 \mathrm{rpm}$.

In addition to these standard behavior constraints on stresses, there are constraints related to the area weighted mean hoop stress (AWMHS) [3] and the area weighted mean radial stress (AWMRS) [3]. These constraints are not readily available in the program like ANSYS and these are the constraints which are used in aero industry. Hence, many non-standard constraints have been incorporated into design rules for the effective evaluation for rotor components.

\subsection{Topological constraints}

DOE is performed by utilizing the simple disc 2-D axis symmetry. By executing DOE an optimum design can be obtained with minimum weight and maximum strength. The design parameters which should be obtained by performing DOE are as shown in figure (4).

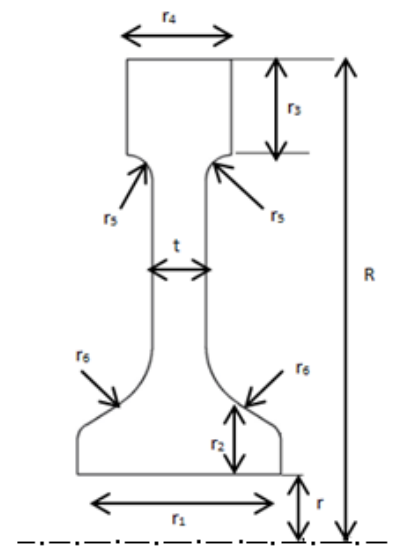

Fig.4 shows the disc parameters

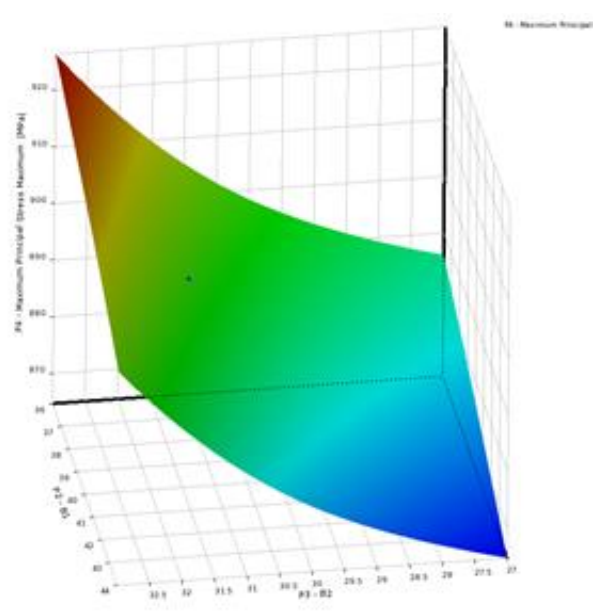

Fig. 5 shows 3-D plot for feasible design points and variations of maximum principle stress

The inner radius ' $r$ ' and outer radius ' $R$ ' of the disc are constant. From the above obtained parameters range, disc can be designed with an optimum dimension which results in less weight and high strength. By using commercial package ANSYS, a non-linear design of experiments is conducted to arrive at feasible possibility in design space. Based on the constraints the optimum design surface recommended meeting the design goal is considered for further analysis in 3-D.

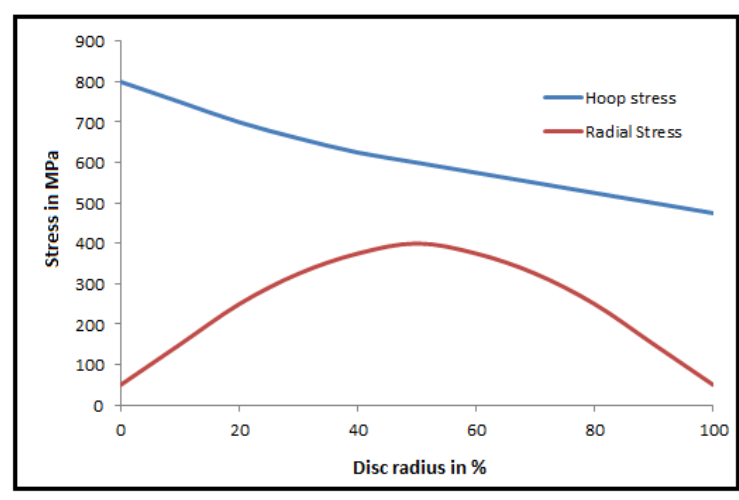

Fig.6 shows variation of stress with respect to the length of the disc sector

The hoop stress (maximum at the disc bore) induced in the disc sector decrease as the length of disc increase from bore to rim. The radial stress (minimum at the bore and maximum in the web) increase in the bore region and decreases in web region as the length increases. Since the hoop stress is concentrated at disc bore and plays a major role in disc failure. Hoop stress considered for evaluating the safety margins.

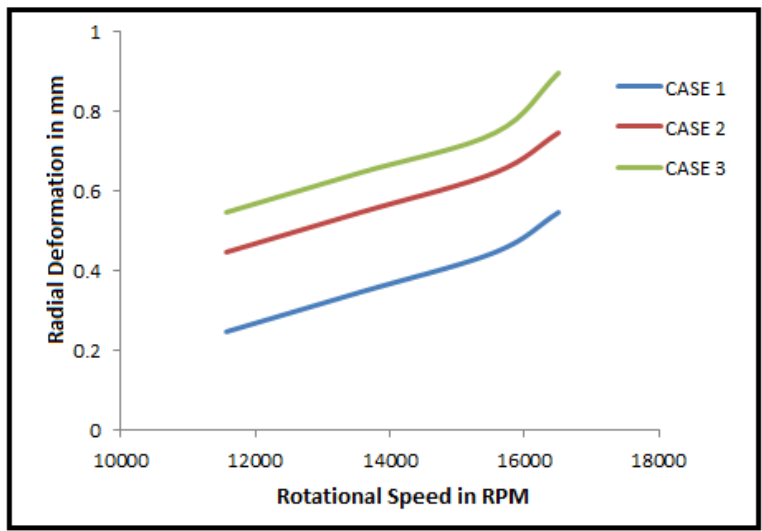

Fig.7 shows the graph plotted for radial growth with change disc speed

The maximum radial gowth of the disc obtained from three cases are less than ' 1 ' which is below the tolerance as mentioned.

\section{Evaluation of over-speed and burst margins}

Over-speed is a condition where the aero engine disc is allowed or forced to rotate beyond its design limit. The consequences of running the disc too fast vary by engine type, model etc. The duration of over-speed is main factor which speed of disc is dependent upon. 
In some aero engine discs, even a momentary of overspeed can result in reducing the engine life or even catastrophic failure. The speed at which disc undergoes catastrophic failure is known as burst speed. In the present work, for evaluating the safety margins and burst speed a 3-D model of a disc section is considered as shown in figure 8.

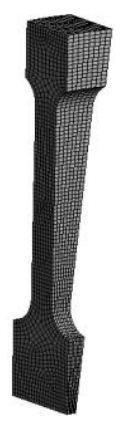

Fig.8 3-D axis- symmetric meshed model using ANSYS

The constraints for rotating speed of aero engine turbine disc given by the two international agencies is given below [EASA CS-E 840 AND FAR 33.27][Stefano Chianese, 2011]

a) $120 \%$ of the maximum permissible rotor speeds associated with any of the ratings of except OEI ratings less than $2 \frac{1}{2}$ minutes.

b) $115 \%$ of the maximum permissible rotor speeds associated with any OEI ratings less than $2 \frac{1}{2}$ minutes.

c) $105 \%$ of the highest rotor speed that would result from the failure of the component or system which is the most critical with respect to over-speeding

d) The burst speed is a highest speed that would result from the failure of any component or system in a respective installation of the engine. This failure of a component or system cannot normally be detected during a routine pre-flight check or during normal flight operation. Gross-yielding approach at critical locations is followed for evaluating the safety margins.

\subsection{Failure criteria for rotating disc}

The determination of the burst-speed has brought to the formulation of several theories and criteria that prescribe the procedure to determine the rotational speed that causes failure. Among them, two are used in this work: the Robinson's Criteria also called average hoop stress criterion; and Hallinan's criteria.

\subsection{Robinson's Criteria [W.N Barack]}

The Robinson criteria is a method which is developed for calculating the burst speed in the hoop mode knowing the ultimate tensile strength, $\sigma$ UTS and the mean hoop stress, $\sigma \mathrm{c}$ mean. As per this criterion burst occurs when the mean hoop stress on a disc section becomes equal to the nominal tensile strength of the material, determined from a uniaxial tensile stress.
The mathematical formulation of Robinson criteria,

$\omega_{\text {burst }}=\omega \sqrt{\frac{\sigma_{U T S}}{\sigma_{c, \text { mean }}}}$

Eqn.3

It is important to know that ultimate strength considered by Robinson Criteria is the engineering stress that differs from true stress. ANSYS applies engineering stress to perform the analysis since; the evolution of safety margins is to be performed by considering true stress. Due to this relation between these stresses is to be performed and utilized for evaluating the safety margins. When passing from engineering stress to true stress, the following relations must be applied:

$\varepsilon_{\text {true }}=\ln \left(1+\varepsilon_{\text {eng }}\right)$

Eqn.4

$\sigma_{\text {true }}=\sigma_{\text {eng }}\left(1+\varepsilon_{\text {eng }}\right)$

Eqn.5

From Eqn. 4 and Eqn. 5 a relationship between true stress and engineering stress was obtained. By utilizing these relations, a graph was plotted between stress and strain which is as shown in figure 9.

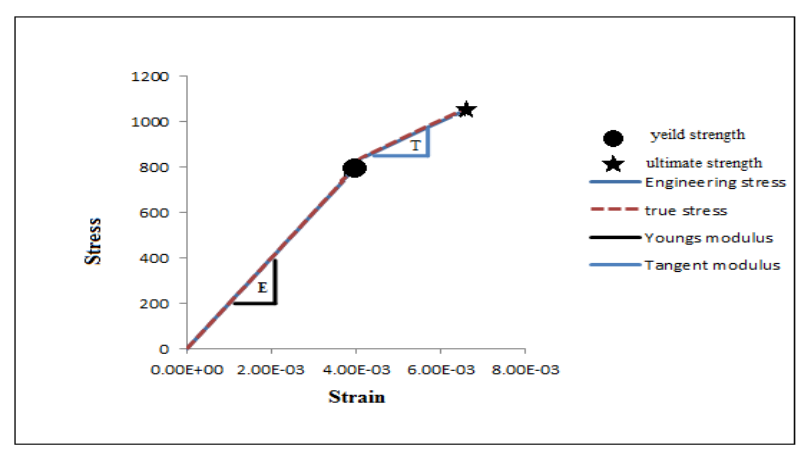

Fig.9 shows the comparison between engginerring stress and true stress

Robinson criteria fail to explain the relation between the true stress and engineering stress. It also fails to explain the effect of maximum hoop stress in the disc failure. Hence this criterion is modified in several ways and one of the forms is Hallinan criteria.

\subsection{Hallinan criteria}

This criteria extends the use of the Robinson criteria considering the maximum hoop stress, $\sigma \mathrm{c}$ max introducing a factor S [W.N Barack] (it is the ratio of true stress to the engineering stress) to weight the influence of the maximum stress over the mean stress. The ratio $S$ is obtained from the graph figure (9) by comparing true and engineering stress.

The mathematical form of Hallinan criteria

$\omega_{\text {burst }}=0.95 \omega\left[S\left(\sqrt{\frac{\sigma_{U T S}}{\sigma_{c, \text { mean }}}}-\sqrt{\frac{\sigma_{U T S}}{\sigma_{c, \text { max }}}}\right)+\sqrt{\frac{\sigma_{U T S}}{\sigma_{c, \text { max }}}}\right] \quad$ Eqn. 6 
These two criterions are similar to each other if the value of $\mathrm{S}$ is close to 1 , such as for discs made of ductile materials.

Therefore, the equation becomes,

$\omega_{\text {burst }}=0.95 \omega \sqrt{\frac{\sigma_{U T S}}{\sigma_{c, \text { mean }}}}$

Eqn. 7

In general, the concept of calculating the average stress is associated with the ductility of material and no difference occurs between the two formulations; while if the disc is made of brittle material it is more appropriate to weight the influence of the maximum stress by applying the Hallinan Formula.

\subsection{Linear Static Analysis}

From the behaviour constraints mentioned over-speed $\geq 118 \%$ of the $100 \%$ speed. Hence, for evaluating the safety margins $121 \%$ speed is considered as per API standards,

Over speed Margin $=\sqrt{\frac{\sigma_{\text {yeild }}}{\sigma_{c, \text { mean }}}}$

Eqn.9

For burst speed,

$\omega_{\text {burst }}=\omega \sqrt{\frac{\sigma_{U T S}}{\sigma_{c, \text { mean }}}}$

Table 2 results for linear static

\begin{tabular}{|c|c|c|c|}
\hline Particulars & Case1 & Case2 & Case3 \\
\hline Over-Speed limits & 1.34 & 1.31 & 1.29 \\
\hline Burst Speed & $190.9 \%$ & $158.7 \%$ & $157.50 \%$ \\
\hline
\end{tabular}

Similarly, for other cases and methods of analysis similar approach is carried out for calculating the overspeed margin, the burst-speed.

Linearity is just an assumption which simplifies the modeling. Every problem is bi-linear, but solving these models time consumption is more when compare to the linear models. Hence, analysis of linear models is carried out whenever possible. However, the assumptions built into linearity must be considered with every model. It is assumed here that the ultimate stress is within the elastic limit and the there is no yield i.e. the stiffness of the material remains constant in linear analysis which is not real. But, the burst takes place in the disc when the induced stress is equivalent to ultimate stress i.e. the linearity of the material is lost. Therefore, the assumptions made are not applicable for evaluating the burst speed. Hence, the burst speed obtained from this approach is in-valid.

\subsection{Bi-linear Isotropic Analysis}

In isotropic hardening when the material is subjected to loading tension or compression the material deforms linearly till the yield limit. Once the material is crossed the yield limit the material doesn't deforms uniformly. But in this case the deformation of the material takes place by maintaining its center constant. This implies that stress while tension and compression remains same in value but opposite in signs, which is not possible in the real behavior of the material. Hence the obtained burst speeds by this approach are also unacceptable.

Table 3 results for bi-linear isotropic

\begin{tabular}{|c|c|c|c|}
\hline Particulars & Case1 & Case2 & Case3 \\
\hline Over-Speed limits & 1.35 & 1.30 & 1.29 \\
\hline Burst Speed & $186.14 \%$ & $153.33 \%$ & $152.73 \%$ \\
\hline
\end{tabular}

\subsection{Bi-linear kinematic Analysis}

The isotropic model implies that, if the yield strength in tension and compression are initially the same, i.e. the yield surface is symmetric about the stress axes; they remain equal as the yield surface develops with plastic strain. Real metals exhibit some isotropic hardening and some kinematic hardening. In Isotropic hardening the material just hardens until it responds elastically. To fix this, alternative laws i.e. kinematic hardening laws have been introduced. As per these hardening laws, the material softens in compression and thus the yield surface remains the same shape and size but merely translates in stress space, which gives the real behavior of the material. Hence, this approach gives the real behavior burst speed obtained are acceptable.

Table 4 results for bi-linear kinematic

\begin{tabular}{|c|c|c|c|}
\hline Particulars & Case1 & Case2 & Case3 \\
\hline $\begin{array}{c}\text { Over-Speed } \\
\text { limits }\end{array}$ & 1.34 & 1.29 & 1.27 \\
\hline Burst Speed & $181.37 \%$ & $150.34 \%$ & $149.75 \%$ \\
\hline
\end{tabular}

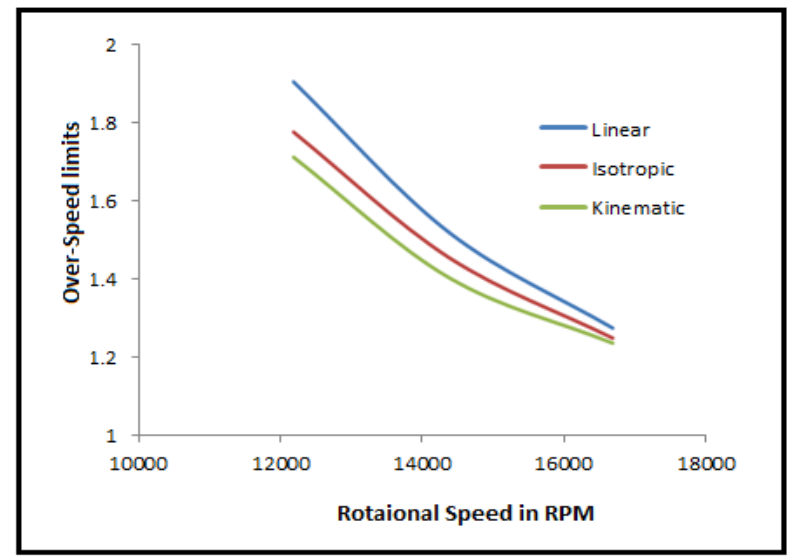

Fig.10 shows OSM for different approachs with respect to change in speed

The evaluated safety margins is plotted in the graph with respect to the change in speed. For over-speed (121\% as per API standards ) condtion the saftey margins obatined for the disc are in the range of 32 $34 \%$ which is as shown in figure (10). 


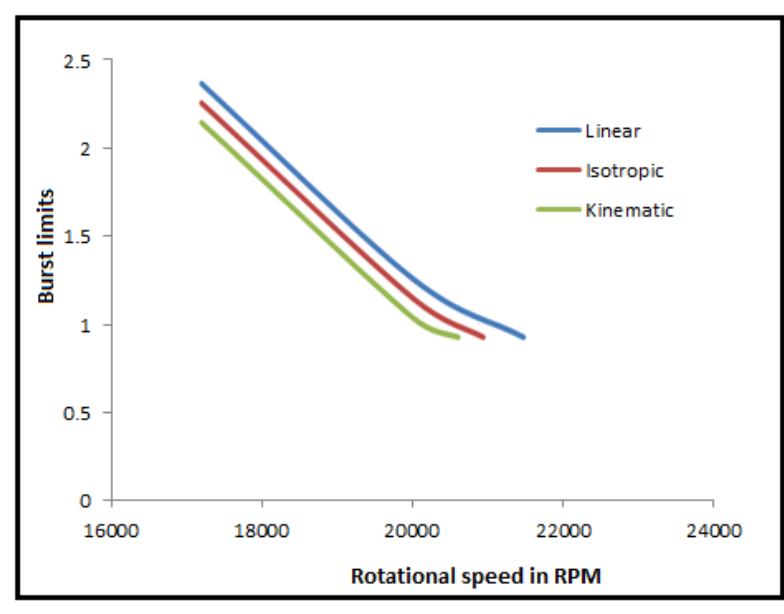

Fig.11 shows burst-margin for different approachs with respect to change in speed

The graph plotted is by considering CASE 3 loading condition. The burst-speed obtained for the disc from different approachs is in the range of 152.73-149.7\% of the operating speed which is as shown in figure (11).

\subsection{Comparison of Burst speed between Robinson and Hallinan criteria}

Hallinan criteria $=0.95^{*}$ Robinson criteria [by using Eqn. (6) \& Eqn. (1)]

Therefore,

Burst speed in Hallinan criteria $=0.95^{*} 190.9 \%=$ $181.355 \%$

Similarly, the above is used to calculate for all other cases.

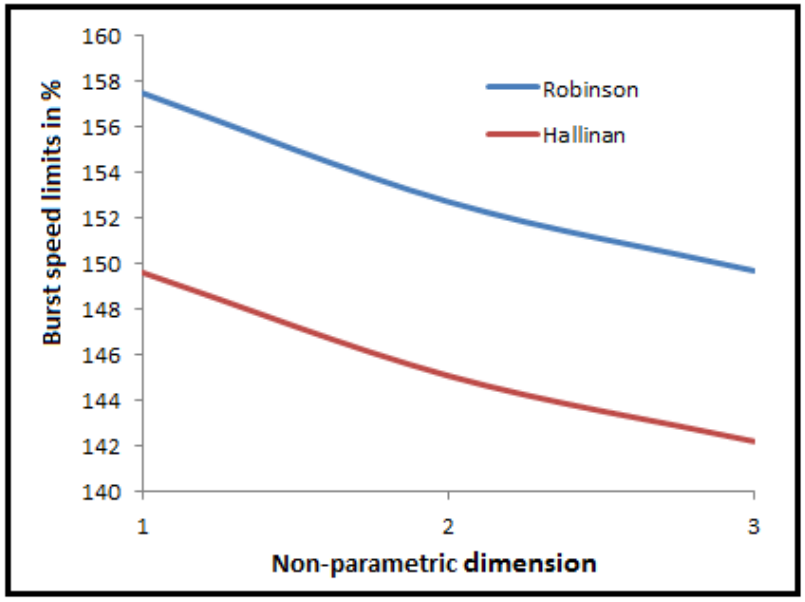

Fig.12 shows burst limits varying with two different approaches

In the above shown figure 1, 2 and 3 represents linear, isotropic, kinematic analysis respectively. It is observed that burst limits obtained from Hallinan is conservative and safe when compare to Robinsons limits.

\section{Fatigue in rotor disc}

ASTM defines fatigue life, Nf, as the number of cycles of specified character that a specimen sustains before failure of a specified nature occurs. In the present work, Universal Slope and Coffin-Manson methods is effectively utilized for estimating the fatigue life of the disc.

\subsection{Estimation of fatigue life}

Estimation of fatigue life cycle is performed by considering the disc at over-speed $(121 \%$ as per API standards) condition

\subsection{Coffin-Manson equation [P. Mestanek,2008]}

This equation gives a relationship between number of cycles and the total strain (elastic + plastic). In mathematical model the equation can be written as,

$\epsilon_{\mathrm{a}}=1.75 \frac{\sigma_{\mathrm{UTS}}}{\mathrm{E}} \mathrm{N}_{\mathrm{f}}^{-.12}+0.5 \epsilon_{\mathrm{f}} \mathrm{N}_{\mathrm{f}}^{-.6}$

Eqn.10

It can be reduced to,

$$
\frac{\epsilon_{\mathrm{a}}}{2}=\epsilon_{\mathrm{f}} 2 \mathbf{N}_{\mathrm{f}}^{c}
$$

\subsection{Universal slope method [U. Muralidharan]}

The USM method can be written in mathematical model is given below,

$$
\frac{\epsilon_{\mathrm{a}}}{2}=0.623\left(\frac{\sigma_{\mathrm{UTS}}}{\mathrm{E}}\right)^{0.832} 2 \mathrm{~N}_{\mathrm{f}}^{-0.12}+0.0196 \epsilon_{\mathrm{f}}^{0.15} 2 \mathrm{~N}_{\mathrm{f}}^{-.56} \quad \text { Eqn } 12
$$

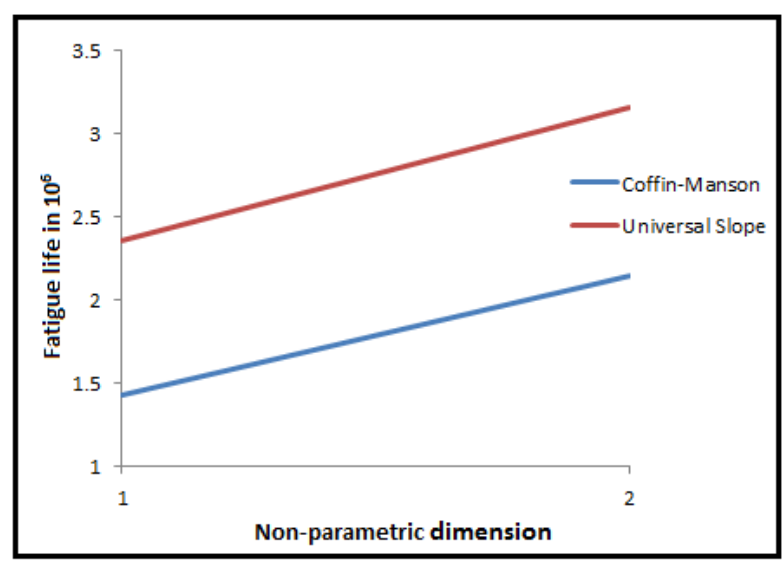

Fig.13 shows low cycle fatigue life varying with two different approaches

In the above figure, 1 and 2 represents linear and bilinear analysis. It is observed that Coffin-Manson method provides a conservative result when compared to universal slope method. 


\section{Conclusions}

- The sensitivity analysis and design checks conducted through blending the classical equations and methodologies has thrown light on various aspects namely design considerations, parametric and design constraints along with behavior constraints to achieve design goal. A nonlinear analysis is must for evaluating the safety limits as per regulations to ensure integrity.

- From the present study for evaluating the safety limits for disc a bi-linear kinematic approach is recommended. The safety limits obtained from this analysis provides a conservative result in a range of $6-8 \%$ when compared to other two approaches

- The burst limits obtained from Hallinans criteria is considered since, the results are 5-7\% conservative when compare to Robinsons criteria. By incorporating this criterion in FE code would help in evaluating burst limits.

- The estimated fatigue life of the disc obtained from Coffin-Manson and Universal Slope method is greater than $1^{*}$ e6 cycles. For, estimating the fatigue life of disc Coffin-Manson method is optimum since, it provides a factor of safety 1.66 when compare to universal slope method. Universal Slope method is utilized for approximation of fatigue life when there is lack of material data.

\section{References}

Lakshman kasina, Raghavan Kotur and Govindaraji Gnanasundaram (2015) Minimum weight design of Aero engine Turbine disks- ASME

Harinath SP (2017) Evaluation of Over-speed, Burst Margin and Estimation of Low-cycle Fatigue Life of an Aero Engine Disc (IJIRAE) Issue 04, Volume 4
K. Kumar, S.L Ajit Prasad and Shivarudraiah (2010) Strength evaluation in turbo machinery Blade disk assembly at constant speed- IJAME

K. Kumar, Ajit Prasad and K Ramachandra (2010), Critical issues in assessment of over speed and burst margin in aero engine discs, International Journal of Computer Applications in Engineering Technology and Sciences, Vol2(1)

S.A Zamani, S.R Tahmasbpour Oman, B.Asadi and M. Hosseinzadh (2014) Numerical simulation and plane stress analytical solution of rotating disk in high speed Indian J.sci res-3

Maruthi B.H, M. Venkatarama Reddy, K. Channakeshavalu (2012) Finite element formulation for prediction of over speed and burst margin limits in Aero- margin IJSCE 2012, 2231-2307, VOL -2

Stefano Chianese (2011) Safety factor against burst speed of turbo machinery rotating discs

W.N Barack and P.A Domas An improved turbine disk design to increase reliability of aircraft jet engines NASA CR135033

S.A.Meguid, P., S Kanth et al. (2002), Finite elements in analysis of Fir-tree region in turbine discs, Finite elements in analysis and design, Vol 35, 3053-317

Gayada, J. and Kantzos, P., (2004), High Temperature Burst testing of Super Alloy Disk with a Dual Grain Structure, NASA/TM-2004-212884

Lucjan Witek (2006) Failure analysis of turbine disc of an aero engine Engineering Failure Analysis 13 9-17

K. L. Singh- and V. R. Ranganath Cycle counting using rain flow algorithm for fatigue analysis- Structural Integrity Division, NAL, Bangalore, India

P. Mestanek (2008) Low cycle fatigue analysis of a last stage steam turbine blade- Applied and Computational Mechanics 2 71-82

U. Muralidharan A Modified Universal Slopes equation for estimation of fatigue characteristics of metals - journal of engineering materials and technology 110(1):55 